\title{
BODY FAT DISTRIBUTION AND METABOLIC CONSEQUENCES - EXAMINATION OPPORTUNITIES IN DOGS
}

\author{
Linda MÜLLER $^{1^{*}}$, Eszzter KOLLÁR ${ }^{1}$, Lajos BALOGH ${ }^{2}$, Zita PÖSTÉNYI ${ }^{2}$, \\ Teréz MÁRIÁN ${ }^{3}$, Ildikó GARAI ${ }^{3}$, László BALKAY ${ }^{3}$, György TRENCSÉNYI ${ }^{3}$ \\ and Julianna THURÓCZY ${ }^{1}$
}

${ }^{1}$ Department and Clinic of Obstetrics and Animal Reproduction, Faculty of Veterinary Science, Szent István University, István u. 2, H-1078 Budapest, Hungary; ${ }^{2}$ National Frédéric Joliot-Curie Institute of Radiobiology and Radiohygiene, Budapest, Hungary; ${ }^{3}$ Department of Nuclear Medicine, Faculty of Medicine, University of Debrecen, Debrecen, Hungary

(Received 19 December 2012; accepted 2 April 2013)

The relationship between metabolic disorders and the distribution of fat in different body regions is not clearly understood in humans. The aim of this study was to develop a suitable method for assessing the regional distribution of fat deposits and their metabolic effects in dogs. Twenty-five dogs were subjected to computed tomographic (CT) imaging and blood sampling in order to characterise their metabolic status. The different fat areas were measured on a cross-sectional scan, and the animals' metabolic status was evaluated by measuring fasting glucose, insulin and leptin levels. The volume of visceral adipose tissue is the main determinant of leptin levels. The correlation of visceral fat volume and leptin concentration was found to be independent of insulin levels or the degree of insulin resistance. There was a positive correlation between the visceral to subcutaneous fat volume ratio and serum insulin concentration, and a similar trend was observed in the relationship of fat ratio and insulin resistance. The distribution of body fat essentially influences the metabolic parameters in dogs, but the effects of adiposity differ between humans and dogs. The findings can facilitate a possible extrapolation of results from animal studies to humans with regard to the metabolic consequences of different obesity types.

Key words: Leptin, insulin resistance, abdominal fat, computed tomography

Obesity is a growing problem in companion animals just like in humans. The development of insulin resistance, dyslipidaemia and other metabolic problems appear to be more closely related to the regional distribution of body fat than simply to adiposity or body weight. Even though the phenotypes of human obesity that represent specific risk factors for metabolic disorders have not been reported in dogs, differences in the metabolic effects of increased visceral or

\footnotetext{
"Corresponding author; E-mail: Muller.Linda@aotk.szie.hu; Phone: 0036 (1) 478-4200
} 
subcutaneous adipose tissue volume have been described (Kabir et al., 2005). The distribution of adipose tissue in dogs is important for understanding how the preferential accumulation of fat into visceral depots instead of subcutaneous ones contributes to the pathologic consequences of obesity.

Over the past few years, dogs have been used as a model of the human metabolic syndrome, and to study the link between visceral adiposity and insulin resistance (Bergman et al., 2001). Although many human studies have shown that different fat depots are quite different metabolically, there is no consensus regarding their relative importance (Dusserre et al., 2000). Previous publications have suggested that excess intraabdominal fat is the major determinant of insulin resistance and of other features of human metabolic syndrome (Fujioka et al., 1987). A study performed in dogs has demonstrated that the surgical excision of omental fat improves insulin sensitivity (Lottati et al., 2009). Other studies reported that the accumulation of abdominal subcutaneous adipose tissue is a better predictor of insulin sensitivity than visceral fat (Goodpaster et al., 1997).

Quantitative estimation of adiposity and fat distribution is essential for examining the effects of obesity. Most methods used in human medicine are not adaptable to dogs because of the variations of their body dimensions. The body condition score (BCS) system is the most commonly used method to diagnose obesity in the dog and to predict the detrimental health effects, but it is based on subjective assessment and does not make it possible to determine the amount of visceral and subcutaneous fat separately (Laflamme, 1997). In the human medical practice, computed tomography (CT) is one of the most advanced methods for the accurate assessment of intraabdominal fat accumulation (Hirooka et al., 2005). Although the CT method using a single image at vertebrae L3-5 is a useful technique for the evaluation of canine obesity and body fat distribution, an accurate procedure has been developed for a single breed (the Beagle) only (Ishioka et al., 2005a).

The adipose tissue is also identified as an endocrine organ that can influence various physiological processes by releasing metabolically active substrates termed adipokines. There are significant differences in the range of adipokine production between preadipocytes and mature adipocytes and between cells localised in different places. Some adipokines (primarily leptin, adiponectin and resistin) have been investigated as potential markers of the degree of obesity and to assess the risk of development of associated complications (German et al., 2010). Serum leptin concentration positively correlates with body fat content, and therefore it is a useful marker for the quantitative assessment of adiposity in dogs, regardless of gender, oestrous cycle, breed and age, although a breed effect on leptin level has not yet been completely excluded. Leptin concentrations were significantly higher in all breeds of dogs with higher BCSs (Ishioka et al., 2002a; Ishioka et al., 2007). Thus, leptin might be a useful factor for the diagnosis of obesity and for assessing the progression of related diseases; moreover, it can be 
used for monitoring fat distribution. Although some researchers have reported that the correlation of leptin levels is the highest with visceral fat volume (Ronnemaa et al., 1997), most studies have shown that leptin concentration is determined mainly by the subcutaneous depot because of a higher secretion rate of leptin (Cnop et al., 2002). Insulin secretion has been suggested to be an additional determinant of leptin concentrations (Malmström et al., 1996), and leptin levels may increase or decrease the glucose-stimulated insulin secretion, depending on the condition of individuals and other metabolic circumstances (Ceddia et al., 2002).

The aim of this study was to examine the effect of fat distribution and the amount of subcutaneous or visceral fat tissue on leptin concentrations, to assess the influence of insulin levels and sensitivity on leptin levels, and to check the possibility of a reverse effect.

\section{Materials and methods}

\section{Animals and general procedures}

The study complied with the requirements of the animal welfare regulations of Szent István University and was conducted in accordance with the European Animal Welfare Regulations. Twenty-five client-owned dogs of different breeds were included in this study. All these dogs were subjected to clinical examinations and to the estimation of adiposity by BCS on the 5-point scale (Edney and Smith, 1986). Detailed data, such as breed, age, gender, body condition and clinical history, were recorded for each dog.

\section{Computed tomography}

Computed tomographic scans (Phillips Brillance, 64 slices CT) of 25 dogs (16 male, 9 female) examined for diagnostic purposes at the Department of Nuclear Medicine of the University of Debrecen (Medical and Health Science Centre) were considered in this study. The dogs were subjected to general anaesthesia for the procedure. Subcutaneous and visceral fat areas were measured on a crosssectional scan $(120 \mathrm{Kv}, 30 \mathrm{~mA}, 5 \mathrm{~mm}$ slice thickness) at the level of the L2 and L3 vertebrae (Ishioka et al., 2005a). The pixels with attenuation values in the selected range were depicted as red (Fig 1). The boundary between visceral and subcutaneous fat tissue was defined by use of abdominal wall musculature in continuity with the deep fascia of the paraspinal muscles. Fat areas were calculated by dividing the number of pixels counted as fat by the ratio of the total number of pixels and known area for a $1 \mathrm{~cm}$ image, and the area of different fat deposits was expressed in square centimetres $\left(\mathrm{cm}^{2}\right)$. Visceral (IAF) or subcutaneous (SCF) fat area percentages were calculated by dividing the fat area by the total 
area in each slice (IAFxL2, IAFxL3, SCFxL2, SCFxL3) and by dividing the fat area with the area of organs (IAForgxL2, IAForgxL3, SCForgxL2, SCForgxL3) to ensure the comparability of data from dogs of different body size.

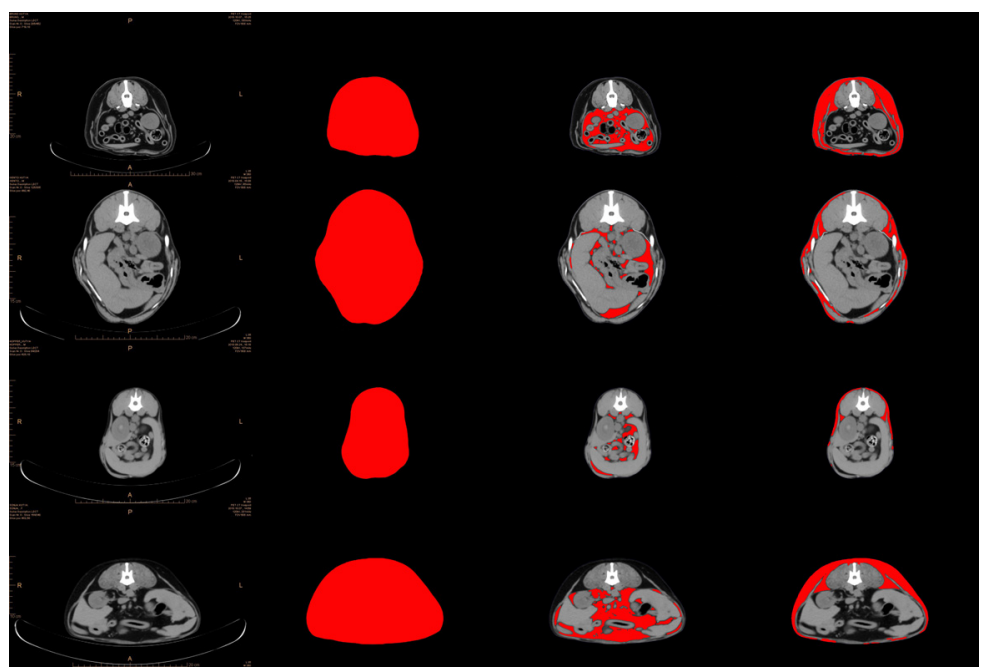

Fig. 1. Computed tomographic (CT) scans. Cross-sectional scans at the level of L2 of four dogs of different breeds and conditions (column 1). CT scans indicating the total area of the body in red (column 2) and the marked area of visceral (column 3) and subcutaneous fat (column 4)

\section{Sample collection and hormone assay}

Blood samples were collected from the cephalic vein before the induction of anaesthesia. Being aware of the effects of diurnal variations, food intake and certain medications on serum leptin concentration (Ishioka et al., 2002 $b$; Ishioka et al., 2005b), we used only blood samples collected from dogs that had been fasted for at least $10 \mathrm{~h}$ and not treated with insulin or glucocorticoids. Blood glucose was measured using an automatic analyzer (Dcont Personal) with a commercial kit, immediately after blood collection. Samples were centrifuged at $1500 \mathrm{rpm}$ for $12 \mathrm{~min}$ at $4{ }^{\circ} \mathrm{C}$, and the serum was separated and chilled until assayed for hormone levels. Hormone determinations were done at the Laboratory of the Department and Clinic of Reproduction, Faculty of Veterinary Science, Szent István University. Leptin concentrations were determined by the use of a direct sandwich ELISA using a goat anti-canine leptin antibody (Canine Leptin ELISA, Millipore Corporation, St. Charles, Missouri). The sensitivity for this assay is $0.4 \mathrm{ng} / \mathrm{ml}$. Insulin levels were measured by using a solid phase two-site ELISA (Mercodia AB, Uppsala, Sweden). It is based on the direct sandwich technique in which two monoclonal antibodies are directed against separate antigenic determinants on the insulin molecule. The detection limit was $0.01 \mu \mathrm{g} / \mathrm{l}$. Numerical results were obtained using an appropriate ELISA reader (Thermo 
Labsystems Multiskan RC, Vantaa, Finland) and a computer programme (Ascent Thermo Labsystem, Vantaa, Finland). Fasting insulin resistance was estimated with Homeostatic Model Assessment (HOMA). HOMA-IR is a simple index of insulin resistance based on fasting glucose and insulin levels (Matthews et al., 1985). This index is calculated as: HOMA-IR $=\{[$ fasting insulin $(\mu \mathrm{U} / \mathrm{ml})] \times$ [fasting glucose $(\mathrm{mmol} / \mathrm{l})]\} / 22.5$.

\section{Statistical analysis}

The data are presented as mean \pm standard deviation (SD) values. Because the variables were not normally distributed, the association between the serum leptin levels and the distribution of abdominal fat tissue, serum insulin level or insulin sensitivity was determined by Spearman's rank order correlation. Simple and multiple regression analyses were used to find out whether changes in leptin levels can explain possible effects of fat distribution, and to evaluate the simultaneous contributions of fat distribution and insulin sensitivity to leptin level. Statistical analyses were made using the $\mathrm{R}$ free software ( $\mathrm{R}$ version 2.9.2, 2009.) A $P$ value of $<0.05$ was considered as statistically significant.

\section{Results}

\section{Condition and fat distribution}

The distribution of dogs according to their BCS was as follows: 17 were normal (BCS3), 7 overweight (BCS4) and 1 obese (BCS5). Sixteen were intact males and nine intact females. The animals were between 2 and 13 years of age (average 8.3). The leptin concentrations were $3.22 \pm 3.74 \mathrm{ng} / \mathrm{ml}$ in BCS3, $10.4 \pm$ 7.36 in BCS4 and $16.06 \mathrm{ng} / \mathrm{ml}$ in BCS5. Leptin levels were significantly higher in dogs with higher BCSs. The insulin level and the HOMA-IR index values tended to decline in dogs with higher BCS categories but there was no significant difference between them. We successfully determined the distribution of abdominal fat in dogs of different breeds, and the area of different fat depots on the CT scans at the level of the L2 and L3 lumbar vertebrae (Fig. 1). The results were expressed in proportion of the total area and as a ratio to the area of abdominal organs.

\section{Effect of fat distribution and insulin concentration on leptin level}

We examined the relationship between the area and the visceral to subcutaneous ratio (IAF/SCFxL2, IAF/SCFxL3) of fat depots as a relative index of intraabdominal fat accumulation (Fujioka et al., 1987), as well as some metabolic parameters. Correlation analysis (Table 1) revealed that there was a significant positive correlation between leptin concentration and the area of subcutaneous 
(0.53-0.61) and visceral fat tissues $(0.78-0.83)$ at the level of L2 and L3. The area of subcutaneous fat showed a lower correlation with serum leptin concentration. Leptin levels did not correlate with the ratio of fat depots, insulin level or HOMA-IR. There was a positive correlation between the intraabdominal to the subcutaneous fat area ratio and insulin concentration, and a similar trend was observed in the relationship of fat ratio with HOMA-IR. The graphic display (Fig. 2) indicates a non-linear relationship between leptin level and the area of visceral fat (IAFxL2). We attempted to examine this relationship using log-transformed leptin concentration values in the regression models. Linear regression analysis showed that leptin concentrations significantly correlated with fat distribution, and revealed that leptin levels could be best predicted from visceral fat (IAForg.xL2, $\mathrm{R}^{2}:$ 64\%; IAFxL2, $\mathrm{R}^{2}: 62 \%$; SCForg.xL2, $\mathrm{R}^{2}: 34 \%$; SCFxL2, $\mathrm{R}^{2}$ : $27 \%$ ), but fat distribution had no significant effect on leptin level. Multiple linear regression analysis indicated that fasting serum insulin and HOMA-IR were not predictors of leptin level; neither these nor the area of subcutaneous fat tissue influenced the relationship between leptin levels and the area of visceral fat.

\section{Table 1}

Spearman's correlations of leptin and insulin levels and HOMA-IR with each other and the parameters of abdominal fat distribution

\begin{tabular}{|c|c|c|c|c|c|c|}
\hline \multirow[b]{2}{*}{$\begin{array}{l}\mathrm{N}=25, \\
{ }^{*} \mathrm{~N}=24\end{array}$} & \multicolumn{2}{|c|}{ Leptin } & \multicolumn{2}{|c|}{ Insulin } & \multicolumn{2}{|c|}{ HOMA-IR } \\
\hline & $P($ rho $)$ & $\mathrm{P}$ & $P$ (rho) & $\mathrm{P}$ & $P$ (rho) & $\mathrm{P}$ \\
\hline IAFxL2 & 0.78 & 0.0000 & 0.22 & 0.3013 & 0.21 & 0.3073 \\
\hline IAFxL3 & 0.80 & 0.0000 & 0.25 & 0.2295 & 0.23 & 0.2786 \\
\hline IAForg.xL2 & 0.79 & 0.0000 & 0.15 & 0.4854 & 0.13 & 0.5235 \\
\hline IAForg.xL3 & 0.83 & 0.0000 & 0.17 & 0.4107 & 0.16 & 0.4515 \\
\hline SCFxL2 & 0.53 & 0.0064 & -0.17 & 0.4225 & -0.17 & 0.4305 \\
\hline SCFxL3 & 0.53 & 0.0062 & -0.21 & 0.3212 & -0.19 & 0.3580 \\
\hline SCForg.xL2 & 0.61 & 0.0011 & -0.15 & 0.4739 & -0.15 & 0.4707 \\
\hline SCForg.xL3 & 0.62 & 0.0009 & -0.13 & 0.5210 & -0.14 & 0.5032 \\
\hline $\begin{array}{l}\text { IAF/ } \\
\text { SCFxL2 }\end{array}$ & $\begin{array}{l}0.08 / \\
0.19^{*}\end{array}$ & $\begin{array}{l}0.7035 / \\
0.3859^{*}\end{array}$ & $\begin{array}{l}0.34 / \\
0.41^{*}\end{array}$ & $\begin{array}{l}0.1000 / \\
0.0476^{*}\end{array}$ & $\begin{array}{l}0.34 / \\
0.39^{*}\end{array}$ & $\begin{array}{l}0.0979 / \\
0.0604^{*}\end{array}$ \\
\hline $\begin{array}{l}\text { IAF/ } \\
\text { SCFxL3 }\end{array}$ & $\begin{array}{l}0.17 / \\
0.28^{*}\end{array}$ & $\begin{array}{l}0.4222 / \\
0.1816^{*}\end{array}$ & $\begin{array}{l}0.36 / \\
0.44^{*}\end{array}$ & $\begin{array}{l}0.0731 / \\
0.0304^{*}\end{array}$ & $\begin{array}{l}0.33 / \\
0.39^{*}\end{array}$ & $\begin{array}{l}0.1041 / \\
0.0608^{*}\end{array}$ \\
\hline Insulin & -0.01 & 0.9734 & & & & \\
\hline HOMA-IR & 0.01 & 0.9735 & & & & \\
\hline
\end{tabular}

$P$ (rho): Spearman's correlation coefficient, ${ }^{*}$ ignoring the outlier value 


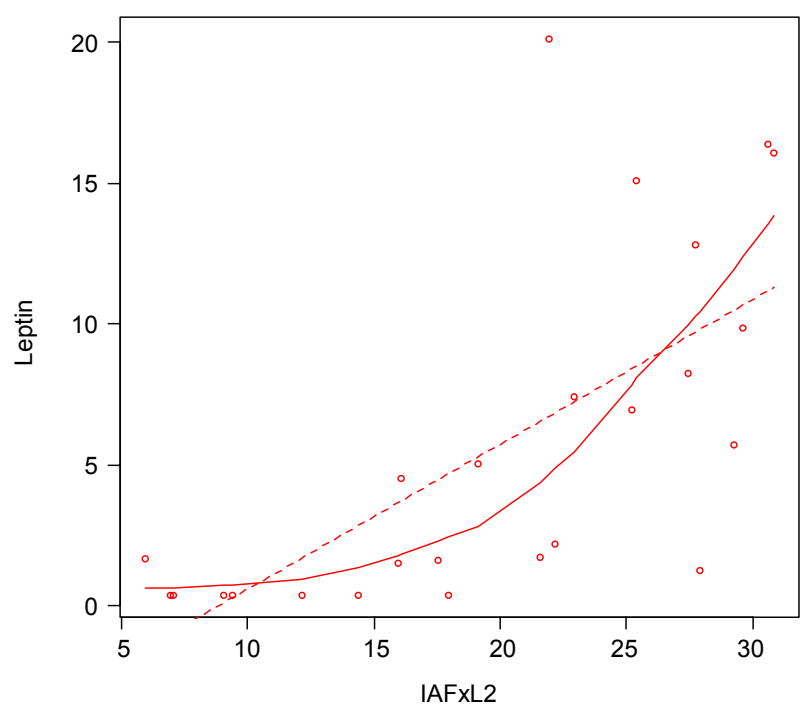

Fig. 2. Relationship between serum leptin and visceral depot (IAFxL2) in dogs. Solid line: lowess nonparametrics regression line; broken line: least-square regression line

\section{Discussion}

\section{Abdominal fat distribution}

The variance in fat deposition is an important similarity between humans and dogs, nevertheless there is no publication about the types of obesity (visceral-subcutaneous, android-gynoid) or about the differences in the distribution of abdominal fat in dogs. Our data showed differences in the regionality of fat deposits. Canine patients had either visceral or subcutaneous type of deposits where the major part of abdominal fat stayed. Although Bergman et al. (2006) reported a tendency for visceral adiposity to increase rapidly as the body fat of dog increases, and also that subsequently the visceral fat accumulation tends to plateau and subcutaneous fat increases more rapidly with overall obesity, we have not found this regularity in our studies. The increase in the amount of visceral fat was not followed by the regular change of abdominal subcutaneous depot. The distribution of abdominal fat tissue varies greatly regardless of the BCS category. However, we can talk about tendencies only since our samples did not cover all BCS categories and the number of cases differed substantially in the different categories. By comparing the results of individuals in BCS3 and BCS4 categories, the greater amount of subcutaneous and visceral fat at the L2 and L3 levels in subjects with higher BCSs could clearly be seen, but an assessment of the ratio of depots by BCS category was not feasible. Based on our results, CT can successfully be applied for the determination of abdominal fat distribution in dogs. 


\section{Abdominal fat distribution and leptin level}

Leptin concentrations were significantly higher in dogs with higher BCSs in our study. Although the standard deviation of the measured leptin levels at each BCS was high (this can be possibly avoided in future examinations by using more objective body condition estimating methods or the 9-point BCS scale), these data were roughly of the same magnitude as data reported in the literature (Ishioka et al., 2007). There was a significant positive correlation between serum leptin concentration and the area of subcutaneous and visceral fat tissues. The amount of visceral fat showed a higher correlation with leptin concentration than that of subcutaneous fat. Overall, leptin concentration is also significantly correlated with abdominal fat distribution in the dog but, unlike in humans, leptin level could best be predicted from the visceral depot. Then again, leptin levels showed no correlation with the fat distribution. These findings are in contradiction with those of human studies, which reported that the leptin secretion rate is higher in subcutaneous than in omental fat tissue, which can be traced back to increased cell size and leptin gene expression (Hube et al., 1996). The higher expression in the falciform ligament may partially explain why the amount of visceral adipose tissue is the main determinant of serum leptin levels in dogs (Ryan et al., 2010). The other factor that can explain the differences is the size of the adipocytes (Wåhlen et al., 2011). In a canine model it has been demonstrated that adipocytes from the visceral depot are larger than subcutaneous fat cells, and that fat feeding increases adipocyte size in both depots; however, while feeding a high fat diet, a modest increase was shown in cell size and number in the subcutaneous fat tissue. Besides the relevant adipocyte enlargement, no hyperplasia was observed in visceral depots (Kabir et al., 2011).

\section{Abdominal fat distribution and insulin sensitivity}

The amount of visceral fat has a strong association with disturbances in insulin action (Cnop et al., 2002). Investigators demonstrated in a dog model that a minimal reduction in visceral fat mass may result in an improvement in insulin sensitivity (Lottati et al., 2009). Other authors have reported that the abdominal subcutaneous fat is the major determinant of insulin sensitivity (Goodpaster et al., 1997). Our findings showing a positive correlation between insulin concentration and abdominal visceral to subcutaneous fat volume ratio are in line with the above results. A similar trend was observed in the relationship of fat ratio with HOMA-IR. These results are consistent with the conclusions of human studies that the intraabdominal to abdominal subcutaneous fat volume ratio was in close relationship with metabolic disorders (Fujioka et al., 1987). Despite this and unlike in many previous communications, insulin level and HOMA-IR value showed no correlation with visceral or subcutaneous adiposity. 


\section{Leptin, insulin and insulin sensitivity}

Leptin inhibits insulin secretion and has anti-insulin effects (Girard, 1997). It has been demonstrated in mouse models that disruption of leptin signalling results in the dysregulation of the insulin secretion and in whole-body insulin resistance (Levi et al., 2011). Leptin might mediate compensatory increases in insulin secretion in obese dogs; however, in contrast to humans, supraphysiological concentrations of leptin do not inhibit insulin secretion in normal lean dogs (Verkest et al., 2011). According to our findings, the insulin level and HOMA-IR index tended to decline in dogs with higher BCS categories. Furthermore, there was a positive correlation between the visceral to subcutaneous fat ratio and insulin concentration. A similar trend was observed in the relationship between the fat ratio and HOMA-IR as well. In spite of this, there was no difference in insulin level or sensitivity between the BCS categories and the leptin concentration did not correlate with insulin level or HOMA-IR. Although insulin also plays an important role in chronic regulation of leptin secretion (Malmström et al., 1996), our results showed that fasting serum insulin and HOMA-IR were not predictors of leptin levels, neither these nor the amount of abdominal subcutaneous fat tissue influenced the relationship between leptin levels and visceral fat volume. Since the standard deviation in insulin concentration and HOMA-IR values increased with increasing visceral fat, it can be concluded that the increase in the amount of fat is not a direct cause of resistance but it predisposes to decreased insulin sensitivity. Regarding obese dogs, two groups should be distinguished. One type is obese dogs with metabolic disorders. The background of this form may also be endocrine abnormalities or existing disturbances of the compensation process, which causes the deterioration of insulin sensitivity or the lack of insulin production. The other type is obesity caused by positive energy balance, when the metabolic balance is maintained despite the saturation of fat depots, deterioration in sensitivity is mild, and only a rise in insulin levels can be detected, which is the result of compensatory mechanisms.

In conclusion, the CT image evaluation method developed in this study may contribute to the clinical diagnosis and the study of canine obesity, especially to studies on the relationship between fat distribution and obesityassociated diseases. According to our results, leptin concentration is correlated with abdominal fat distribution, and it could appropriately be predicted from the amount of visceral fat. Unlike in humans, the deposited visceral fat is the main determinant of leptin levels in dogs, while the abdominal subcutaneous fat has little effect. Leptin levels, however, show no correlation with the ratio of depots. In contrast to the recently published assumptions, the correspondence of visceral fat and leptin concentration is not significantly affected by serum insulin levels or the degree of insulin resistance. On this basis, serum leptin levels may be a useful marker in analyses of abdominal body fat distribution in dogs. It is important to take into account the differences between humans and dogs. The insulin 
level and HOMA-IR index tend to decline in dogs with higher BCS categories. There is a positive correlation between intraabdominal fat to abdominal subcutaneous fat ratio and insulin concentration, and a similar trend can be observed in the relationship of fat ratio with HOMA-IR. Further histopathological works to measure fat cell size as well as leptin-receptor immunohistochemistry and blood chemistry are in progress to facilitate a better understanding of the effects of fat depots and their regionality in dogs.

\section{Acknowledgements}

The study was sponsored by the TÁMOP-4.2.2.B-10/1 and TÁMOP-4.2.1.B-11/2/ KMR-2011-0003 projects. We are indebted to Veronika Kovács-Haász, Tamás Nagy and Judit Szabó for their technical help. Special thanks are due to Andrea Harnos for her help in with the statistical analysis of data and to Anna Wölfling for her excellent technical help with the ELISA assays.

\section{References}

Bergman, R. N., Kim, S. P., Catalano, K. J., Hsu, I. R., Chiu, J. D., Kabir, M., Hucking, K. and Ader, M. (2006): Why visceral fat is bad: mechanisms of the metabolic syndrome. Obesity 14, 16S-19S.

Bergman, R. N., Van Citters, G. W., Mittelman, S. D., Dea, M. K., Hamilton-Wessler, M., Kim, S. P. and Ellmerer, M. (2001): Central role of the adipocyte in the metabolic syndrome. J. Invest. Med. 49, 119-126.

Ceddia, R. B., Koistinen, H. A., Zierath, J. R. and Sweeney, G. (2002): Analysis of paradoxical observations on the association between leptin and insulin resistance. FASEB J. 16, 1163-1176.

Cnop, M., Landchild, M. J., Vidal, J., Havel, P. J., Knowles, N. G., Carr, D. R., Wang, F., Hull, R. L., Boyko, E. J., Retzlaff, B. M., Walden, C. E., Knopp, R. H. and Kahn, S. E. (2002): The concurrent accumulation of intra-abdominal and subcutaneous fat explains the association between insulin resistance and plasma leptin concentrations: distinct metabolic effects of two fat compartments. Diabetes 51, 1005-1015.

Dusserre, E., Moulin, P. and Vidal, H. (2000): Differences in mRNA expression of the proteins secreted by the adipocytes in human subcutaneous and visceral adipose tissues. Biochim. Biophys. Acta 1500, 88-96.

Edney, A. T. B. and Smith, P. M. (1986): Study of obesity in dogs visiting veterinary practices in the United Kingdom. Vet. Rec. 118, 391-396.

Fujioka, S., Matsuzawa, Y., Tokunaga, K. and Tarui, S. (1987): Contribution of intra-abdominal fat accumulation to the impairment of glucose and lipid metabolism in human obesity. Metabolism 36, 54-59.

German, A. J., Ryan, V. H., German, A. C., Wood, I. S. and Trayhurn, P. (2010): Obesity, its associated disorders and the role of inflammatory adipokines in companion animals. Vet. J. 185, 4-9.

Girard, J. (1997): Is leptin the link between obesity and insulin resistance? Diabetes Metab. 3, 16-24.

Goodpaster, B. H., Thaete, F. L., Simoneau, J. A. and Kelley, D. E. (1997): Subcutaneous abdominal fat and thigh muscle composition predict insulin sensitivity independently of visceral fat. Diabetes 46, 1579-1585. 
Hirooka, M., Kumagi, T., Kurose, K., Nakanishi, S., Michitaka, B., Horiike, N. and Onji, M. (2005): A technique for the measurement of visceral fat by ultrasonography: comparison of measurements by ultrasonography and computed tomography. Int. Med. 44, 791-799.

Hube, F., Lietz, U., Igel, M., Jensen, P. B., Tornqvist, H., Joost, H. G. and Hauner, H. (1996): Difference in leptin mRNA levels between omental and subcutaneous abdominal adipose tissue from obese humans. Horm. Metab. Res. 28, 690-693.

Ishioka, K., Hatai, H., Komabayashi, K., Soliman, M. M., Shibata, H., Honjoh, T., Kimura, K. and Saito, M. (2005b): Diurnal variations of serum leptin in dogs: effects of fasting and refeeding. Vet. J. 169, 85-90.

Ishioka, K., Hosoya, K., Kitagawa, H., Shibata, H., Honjoh, T., Kimura, K. and Saito, M. (2007): Plasma leptin concentration in dogs: effects of body condition score, age, gender and breeds. Res. Vet. Sci. 82, 11-15.

Ishioka, K., Okumura, M., Sagawa, M., Nakadomo, F., Kimura, K. and Saito, M. (2005a): Computed tomographic assessment of body fat in beagles. Vet. Radiol. Ultrasound 46, 49-53.

Ishioka, K., Soliman, M. M., Honjoh, T., Shibata, H., Kimura, K. and Saito, M. (2002b): Dexamethasone increases serum leptin concentration in dogs. Vet. J. 164, 295-297.

Ishioka, K., Soliman, M. M., Sagawa, M. M., Nakadomo, F., Shibata, H., Honjoh, T., Hashimoto, A., Kitamura, H., Kimura, K. and Saito, M. (2002a): Experimental and clinical studies on plasma leptin in obese dogs. J. Vet. Med. Sci. 64, 394-353.

Kabir, M., Catalano, K. J., Ananthnarayan, S., Kim, S. P., Van Citters, G. W., Dea, M. K. and Bergman, R. N. (2005): Molecular evidence supporting the portal theory: a causative link between visceral adiposity and hepatic insulin resistance. Am. J. Physiol. Endocrinol. Metab. 288, 454-461.

Kabir, M., Stefanovski, D., Hsu, I. R., Iyer, M., Woolcott, O. O., Zheng, D., Catalano, K. J., Chiu, J. D., Kim, S. P., Harrison, L. N., Ionut, V., Lottati, M., Bergman, R. N. and Richey, J. M. (2011): Large size cells in the visceral adipose depot predict insulin resistance in the canine model obesity. Obesity 19, 2121-2129.

Laflamme, D. (1997): A clinical tool - development and validation of a body condition score system for dogs. Canine Pract. 22, 10-15.

Levi, J., Gray, S. L., Speck, M., Huynh, F. K., Babich, S. L., Gibson, W. T. and Kieffer, T. J. (2011): Acute disruption of leptin signaling in vivo leads to increased insulin levels and insulin resistance. Endocrinology 152, 3385-3395.

Lottati, M., Kolka, C. M., Stefanovski, D., Kirkman, E. L. and Bergman, R. N. (2009): Greater omentectomy improves insulin sensitivity in nonobese dogs. Obesity 17, 674-680.

Malmström, R., Taskinen, M. R., Karonen, S. L. and Yki-Järvinen, H. (1996): Insulin increases plasma leptin concentrations in normal subjects and patients with NIDDM. Diabetologia 39, 993-996.

Matthews, D. R., Hosker, J. P., Rudenski, A. S., Naylor, B. A., Treacher, D. F. and Turner, R. C. (1985): Homeostasis model assessment: insulin resistance and beta-cell function from fasting plasma glucose and insulin concentrations in man. Diabetologia 28, 412-419.

Ronnemaa, T., Karonen, S. L., Rissanen, A., Koskenvuo, M. and Koivisto, V. A. (1997): Relation between plasma leptin levels and measures of body fat in identical twins discordant for obesity. Ann. Intern. Med. 126, 26-31.

Ryan, V. H., German, A. J., Wood, I. S., Hunter, L., Morris, P. and Trayhurn, P. (2010): Adipokine expression and secretion by canine adipocytes: stimulation of inflammatory adipokine production by LPS and TNF $\alpha$. Pflügers Arch. Eur. J. Physiol. 460, 603-616.

Verkest, K. R., Fleeman, L. M., Morton, J. M., Ishioka, K. and Rand, J. S. (2011): Compensation for obesity-induced insulin resistance in dogs: assessment of the effects of leptin, adiponectin, and glucagon-like peptide-1 using path analysis. Dom. Anim. Endocrinol. 41, 24-34.

Wåhlen, K., Sjölin, E. and Löfgren, P. (2011): Role of fat cell size for plasma leptin in a large population based sample. Exp. Clin. Endocrinol. Diabetes 119, 291-294. 